\title{
Future computing with loT and cloud computing
}

\author{
D. S. Park ${ }^{1}$
}

(c) Springer Science+Business Media, LLC, part of Springer Nature 2018

\section{Introduction}

Future computing (FC) is a technology of real-world Internet of things on cloud computing with respect to IT convergence that has emerged rapidly as an exciting new industry and life paradigm. Future computing is being used to encompass the cloud, big data, and cloud data centers that are the megatrends of the computing industry. This technology is making a new future market that is different from the past and is evolving toward gradually eroding the existing market. Internet of things is a vast and dynamic territory and is evolving at a rapid pace. The realization of the Internet of things vision brings ICT technologies closer to many aspects of real-world facing serious issues such as global warming, environment protection, and energy saving on cloud computing. Advanced technologies in sensing, processing, communication, and services are leading to IoT service in our life such as industry, army, and life paradigms on cloud computing environment. There might be many issues to realize and provide smart services, and much effort and enormous attention have been focused on Internet of things on cloud computing technologies. Theoretical research contributions presenting advanced technologies, concepts, or analyses, reports on experiences and experiments of implementation and application of theories, and tutorials on new trends should be needed on this research fields. For the aforementioned reasons, the special issue intends to give an overview of the state of the art of issues and solution guidelines for the FC services with IT convergence.

\section{Related works}

Meng et al. [1] proposed a dynamic multi-constraint service-aware collaborative access algorithm (DCSACA) based on dynamic load feedback to solve the problem of a large number of service request access in DACIOT. DCSACA builds a dynamic context

\footnotetext{
$\triangle \quad$ D. S. Park

parkds2013@gmail.com

1 Soonchunhyang University, Ansan, Republic of Korea
} 
load sensing model (DCLSM) and uses a heuristic down search algorithm. It shows better access performance and better stability compared with the other algorithms.

Kumari et al. [2] proposed an authentication scheme based on ECC for IoT and cloud servers. The authors analyzed the security properties of the proposed scheme by using automated validation of Internet security protocols and applications (AVISPA) tool. The experimental results show that the proposed scheme is invincible to various attacks and is well crafted for IoT and cloud server environment.

Muñoz et al. [3] analyzed the capabilities and weaknesses of penetration and dynamic analysis tools to gain accurate knowledge of these tools. The results of analysis show that the tools need to be configured to the target application to improve accuracy and to reduce the amount of exploited vulnerabilities.

Aslanpour et al. [4] proposed an autonomic resource provisioning mechanism with resource-aware, SLA-aware, and user behavior-aware features to handle resource overprovisioning or under-provisioning problems. In this manuscript, the authors provide providence and flexibility features by using radial basis function neural network. The experimental results show that the proposed mechanism reduces the cost and guarantees the quality of service.

Baek et al. [5] proposed an energy efficiency grading system for mobile applications to handle a problem (the absence of the power-related information of the mobile applications). The evaluation of this system shows that it can help the user to select a better application among exchangeable applications because this system provides the power-related information of the applications.

Kim et al. [6] proposed schemes of the design and implantation of IT convergence framework for games as a service of IoT using lego-typed sensor module. This paper showed the possibilities of games in the IoT environment using lego-typed sensor module by creating games and measuring the interactions of users in the IoT environment.

Chen et al. [7] proposed the probability-based adaptive probabilistic algorithm (APA) to consider the cloud workflow scheduling problem. APS consisted of a new schedule generation scheme (keeping the good blocks to the next generation) and improvement procedure (finding good building blocks). Experimental results demonstrated that APA outperforms the other three adapted algorithms for resource allocation in cloud computing.

Song et al. [8] proposed a method based on the long short-term memory model to predict the mean load over consecutive future time intervals and actual load multi-step ahead. Experimental results in the traditional grid system and cloud environment show that the LSTM model performed a well-adaptive capability and achieves state-of-theart results in both datasets.

Chaabouni et al. [9] provided a guideline by defining and discussing the main important elements that should be considered when building an energy management strategy to optimize energy consumption. This is based on the median absolute deviation method (MAD). Simulation results show better results of the proposed technique compared with the existing ones, especially in reducing energy consumption and the number of virtual machine migrations.

Muñoz et al. [10] conducted an analysis and assessment of vulnerable Web application to evaluate the strengths and weaknesses of tools that detect the vulnerabilities 
of a Web application. In this paper, an analysis of the different vulnerable Web applications is presented. Experimental results show that vulnerable Web applications can help to determine the real detection capabilities of Web analysis tools and to provide security training to developers.

Kim et al. [11] proposed and develop the mobile forensic reference set (MFReS) for mobile forensic issue. The MFReS consists of two repositories as file servers, three reference datasets (RDS) as database servers, and the representational state transfer (REST) that enables the contents of the RDS. This system seems to be helpful in future digital forensic studies on IoT mobile devices.

Wang et al. [12] proposed an improved ACO algorithm approach for WSNs that use mobile sinks by considering $\mathrm{CH}$ distances to find an optimal mobility trajectory. The experimental results show that the proposed approach performs particularly well compared with other standard algorithms. This approach extended the wireless sensor network lifetime.

Lee et al. [13] proposed a mobile device structure to secure the mobile device user information with data privacy and execution privacy. This proposed structure consists of secure service APIs, security functions through SAL, a crypto library, secure storage, key management, inter-domain communication, etc. The experimental results show that the proposed structure has less computing power, data protection stored in mobile device, and secure execution environment.

Ok et al. [14] proposed a modified adaptive support weight scheme and a robust disparity search range estimation scheme. A modified adaptive support weight scheme provides rectangular ring-type window configurations to minimize hardware resources while maintaining matching accuracy. A robust disparity search range estimation scheme uses the stretched depth histograms to reduce the computational overhead of window-based local stereo matching algorithms. Experimental results show that the proposed schemes reduced hardware resources and computational overhead than conventional method.

Kim et al. [15] introduced the application of a text mining analysis technique (three analytical techniques) to identify the knowledge structures of academic research in the field of telecommunications policy and to pinpoint future research opportunities. Three analytical techniques were employed: a productivity analysis; a contents analysis based on topic modeling and word co-occurrence; and an author co-citation analysis based on a hierarchical clustering algorithm, multidimensional scaling, and a factor analysis. The results show that the journal 'Telecommunications Policy' has greatly contributed to the publication and the telecommunication policy studies are undertaken by experts in various research fields.

Cho et al. [16] proposed a method that detects damaged files using information entropy graphs to consider the limitations of the forensic processes for detection and distinction of files. The experimental results show that the proposed method enables quantitative comparison of the similarity of files before and after damage with their original versions through graph pattern similarity test even when files had damaged header, footer, or body regions, the similarity of the graph pattern was preserved.

Oh et al. [17] presented compact deep neural network-based face recognition method for visual Internet of things to solve a problem that current state-of-the-art face recognition methods based on huge deep neural networks are difficult to apply in the 
embedded platform. The experimental results show that the proposed method performs competitive accuracy and performance results on the LFW verification benchmark. Also, the proposed method shows promising results using a mobile face recognition dataset and runs in real time in a mobile embedded environment despite using deep neural network.

Kim et al. [18] proposed a virtual convergence network environment combining both SD-WAN infrastructure and distributed cloud computing resources. The proposed environment presents the secure isolation of logical group networks that are designed to offer very high data transmission performance and automated resource convergence.

Oh et al. [19] proposed a deep feature learning framework for automated large-scale person re-identification with low computational cost and memory usage to find the same individual across cameras in disjoint views at different locations and times. The proposed framework presents the state of the art on two public benchmark datasets (CUHK03 and Market 1501).

Chunxiz et al. [20] proposed an energy-saving strategy based on multi-server vacation queuing theory that switches servers between on and sleep in groups optimal energy use. The experimental results show that the proposed approach is valuable for studying the power performance trade-off in cloud data centers.

Kim et al. [21] proposed a new method in an ambulatory ECG signal in semireal time by implementing the low-order Chebyshev type-II infinite impulse response low-pass filter (IIR-LPF) while compensating its inherent nonlinear-phase response to remove baseline drift that required a considerable amount of off-line computations and suffered from a certain amount of time delay. The experimental results show that the cancelation of baseline fluctuations can be performed by semi-real-time computation.

Li et al. [22] proposed an authentication scheme based on a dynamic multi-key game for access to the devices in a sensor-cloud network using the probability of interception of the key and the leak probability of devices' operating authorization. The proposed approach successfully presents an improvement in the security of IWSC.

Zhu et al. [23] proposed an adaptive multi-objective optimization framework for a multi-source multicasting two-hop multi-input multi-output (MIMO) amplify-forward (AF) relay system to minimize the transmission power consumption and the meansquared error (MSE) of the receiver estimated signal simultaneously. The simulation results show that the proposed algorithm is effective.

Borujeni et al. [24] proposed fog-based energy-efficient routing protocol based on P-SEP which uses FECR and FEAR algorithms to improve the performance of fogsupported WSNs and prolong the lifetime of networks. The simulation results show that the average amount of energy usage in FECR protocol has been reduced and the number of live nodes saved in the network increased in proposed protocols than P-SEP protocol.

Lee et al. [25] proposed an IoT service classification and clustering system to optimize communication and interaction among heterogeneous devices and services. This paper finds five clusters by surveying over 100 commercial IoT services and shows that such services are well clustered by similarity and purpose.

Yang et al. [26] proposed an autonomic resource provisioning mechanism with resource-aware, SLA-aware, and user behavior-aware features to handle resource overprovisioning or under-provisioning problems. In this manuscript, the authors provide 
providence and flexibility features by using radial basis function neural network. The experimental results show that the proposed mechanism reduces the cost and guarantees the quality of service.

Lee et al. [27] provided a way to accelerate existing tasks normally performed by CPUs to improve application and overall system performance and resource utilization without changing or rewriting application code using integrated GPU. The experimental results show the improvement in the effectiveness of using GPU offloading and caching for the bulk memory operations.

Wang et al. [28] analyzed the principles and processes of planning recognition that focused on analyzing the current behavior and abstraction that occurred, which were the process of reasoning with agencies. The results show that this model could increase the efficiency and reliability of plan recognition and provide a vivid description of trend forecasts. The experimental results show that the proposed model could increase the efficiency and reliability of plan recognition and provide a vivid description of trend forecasts.

Linh et al. [29] introduced a transmission method based on HEVC multi-view adaptive video streaming over P2P network in NFV. Voronoi neighbor discovery in $\mathrm{P} 2 \mathrm{P}$ streaming is utilized to find the nearest neighbor in the P2P network.

Jung et al. [30] developed a Web-based task monitoring tool that can check the status of solver results and tasks for analyzing products and monitoring work through CalculiX, an open-source-based finite element analysis (FEA) solver. The experimental results show the performance variation profiling according to the number of CPUs is conducted by running the target application with openMP customization.

Li et al. [31] proposed a trajectory-based crowdsourcing data management method for privacy-aware spatial crowdsourcing using 3DES encryption and a compressionsensing-based trajectory data decryption method called DES-Travec cryptography algorithms.

Park el al. [32] proposed a lightweight PKI (LW PKI) that detects the fake VNFs in a virtual environment and guarantees data security through mutual authentication between VNFs. The experimental results show that the LW PKI is an efficient secure mechanism in a virtual environment, and authentication can be performed without overhead affecting system performance.

Yao et al. [33] proposed a multi-objective transition optimization model based on the method of power function considering both fair and efficiency. The results show that the proposed model has superior performance than classical general smooth transition method in the scenario of evening peak.

Kim et al. [34] proposed a new container fog node-based cloud orchestration which is a prototype of a network management with a lighter container technology than virtualization. The proposed architecture increases the throughput of data in the IoT network, improves performance by providing data quickly, and enables rapid detection of various external attacks through real-time management. 


\section{References}

1. Meng Y, QingKui C (2018) DCSACA: distributed constraint service-aware collaborative access algorithm based on large-scale access to the internet of things. J Supercomput. https://doi.org/10.1007/s1 1227-018-2243-7

2. Kumari S, Karuppiah M, Das AK, Li X, Wu F, Kumar N (2017) A secure authentication scheme based on elliptic curve cryptography for IoT and cloud servers. J Supercomput. https://doi.org/10.1007/s11 227-017-2048-0

3. Muñoz FR, Vega EAA, Villalba LJG (2016) Analyzing the traffic of penetration testing tools with an IDS. J Supercomput. https://doi.org/10.1007/s11227-016-1920-7

4. Aslanpour MS, Dashti SE, Ghobaei-Arani M, Rahmanian AA (2017) Resource provisioning for cloud applications: a 3-D, provident and flexible approach. J Supercomput. https://doi.org/10.1007/s11227017-2156-X

5. Baek D, Park JH, Lee JW (2018) An energy efficiency grading system for mobile applications based on usage patterns. J Supercomput. https://doi.org/10.1007/s11227-018-2439-X

6. Kim HY (2017) A design and implementation of a framework for games in IoT. J Supercomput. https:// doi.org/10.1007/s11227-017-1973-2

7. Chen L, Li X (2017) Cloud workflow scheduling with hybrid resource provisioning. J Supercomput. https://doi.org/10.1007/s11227-017-2043-5

8. Song B, Yu Y, Zhou Y, Wang Z, Du S (2017) Host load prediction with long short-term memory in cloud computing. J Supercomput. https://doi.org/10.1007/s11227-017-2044-4

9. Chaabouni T, Khemakhem M (2017) Energy management strategy in cloud computing: a perspective study. J Supercomput. https://doi.org/10.1007/s11227-017-2154-Z

10. Muñoz FR, Cortes IIS, Villalba LJG (2017) Enlargement of vulnerable web applications for testing. J Supercomput. https://doi.org/10.1007/s11227-017-1981-2

11. Kim D, Lee Y, Lee S (2017) MFReS (mobile forensic reference set) and mobile forensic investigation for android devices. J Supercomput. https://doi.org/10.1007/s11227-017-2205-5

12. Wang J, Cao J, Sherratt RS, Park JH (2017) An improved ant colony optimization based approach with mobile sink for wireless sensor networks. J Supercomput. https://doi.org/10.1007/s11227-017-2115-6

13. Lee YK, Kim JN, Lim KS, Yoon H (2017) Secure mobile device structure for trust IoT. J Supercomput. https://doi.org/10.1007/s11227-017-2155-y

14. Ok SH, Shim JH, Moon B (2017) Modified adaptive support weight and disparity search range estimation schemes for stereo matching processors. J Supercomput. https://doi.org/10.1007/s11227-0172058-y

15. Kim SK, Oh J (2017) Information science techniques for investigating research areas: a case study in telecommunications policy. J Supercomput. https://doi.org/10.1007/s11227-017-2062-2

16. Cho C, Chung K, Won Y (2017) Detection of damaged files and measurement of similarity to originals using entropy graph characteristics. J Supercomput. https://doi.org/10.1007/s11227-017-2121-8

17. Oh SH, Kim GW, Lim KS (2017) Compact deep learned feature based face recognition for visual internet of things. J Supercomput. https://doi.org/10.1007/s11227-017-2198-0

18. Kim D, Kim YH, Kim KH et al (2018) Logically isolated group network for virtual convergence environment over SD-WAN. J Supercomput. https://doi.org/10.1007/s11227-018-2282-0

19. Oh SH, Han SW, Choi BS et al (2018) Deep feature learning for person re-identification in a large-scale crowdsourced environment. J Supercomput. https://doi.org/10.1007/s11227-017-2221-5

20. Chunxia Y, Shunfu J (2018) An energy-saving strategy based on multi-server vacation queuing theory in cloud data center. J Supercomput. https://doi.org/10.1007/s11227-018-2513-4

21. Kim J-H, Lee K-H, Lee J-W Lee, Kim K-S (2018) Semi-real-time removal of baseline fluctuations in electrocardiogram (ECG) signals by an infinite impulse response low-pass filter (IIR-LPF). J Supercomput. https://doi.org/10.1007/s11227-018-2608-y

22. Li D, Li M, Liu J (2018) A dynamic multiple-keys game-based industrial wireless sensor-cloud authentication scheme. J Supercomput. https://doi.org/10.1007/s11227-018-2441-3

23. Zhu M, Zhang D, Wang J (2018) Multi-objective optimization design for multi-source multicasting MIMO AF relay systems. J Supercomput. https://doi.org/10.1007/s11227-018-2275-Z

24. Borujeni EM, Rahbari D, Nickray M (2018) Fog-based energy-efficient routing protocol for wireless sensor networks. J Supercomput. https://doi.org/10.1007/s11227-018-2514-3

25. Lee D, Lee H (2018) IoT service classification and clustering for integration of IoT service platforms. J Supercomput. https://doi.org/10.1007/s11227-018-2288-7 
26. Yang C-T, Chen S-T, Lo Y-S, Kristiani E, Chan Y-W (2018) On construction of a virtual GPU cluster with InfiniBand and $10 \mathrm{~Gb}$ ethernet virtualization. J Supercomput. https://doi.org/10.1007/s11227-01 8-2484-5

27. Lee JH, Shi W, Gil JM (2018) Accelerated bulk memory operations on heterogeneous multi-core systems. J Supercomput. https://doi.org/10.1007/s11227-018-2589-X

28. Wang X, Wang L, Wang J, Li S (2018) An event-driven plan recognition algorithm. J Supercomput. https://doi.org/10.1007/s11227-018-2650-9

29. Van Ma L, Yu G, Kim J-Y, Won Y, Kim J (2018) An efficient transmission method based on HEVC multi-view adaptive video streaming over P2P network in NFV. J Supercomput 1-21. https://doi.org/ 10.1007/s11227-018-2594-0

30. Jung D, Kim M, Lee J, Kim H-Y, Lee D (2018) Job submission and monitoring management in integrated computing environment for finite element analysis. J Supercomput. https://doi.org/10.100 7/s11227-018-2590-4

31. Li Y, Shin B-S (2018) Privacy-aware task data management using TPR*-tree for trajectory-based crowdsourcing. J Supercomput. https://doi.org/10.1007/s11227-018-2486-3

32. Park S, Kim H, Ryou J (2018) Utilizing a lightweight PKI mechanism to guarantee a secure service in a cloud environment. J Supercomput. https://doi.org/10.1007/s11227-018-2506-3

33. Yao J, Zhang K, Dai Y, Wang J (2018) Power function based signal recovery transition optimization model of emergency traffic. J Supercomput. https://doi.org/10.1007/s11227-018-2596-y

34. Kim NY, Ryu JH, Kwon BW, Pan Y, Park JH (2018) CF-CloudOrch: container fog node-based cloud orchestration for IoT networks. J Supercomput. https://doi.org/10.1007/s11227-018-2493-4 